\title{
HÉRNIA PULMONAR INTERCOSTAL: RELATO DE CASO E REVISÃO DE LITERATURA
}

\section{INTERCOSTAL PULMONARY HERNIA: CASE REPORT AND LITERATURE REVIEW}

Marcus da Matta Abreu ${ }^{1}$; Juliana Dias Nascimento Ferreira ${ }^{1}$; Gabriel Ferreira Rezende ${ }^{2}$; Lia

Monnerat Lemos dos Santos².

\section{RESUMO}

A ocorrência de hérnia pulmonar é infrequente. Segundo Morel-Lavalle, elas podem ser classificadas, de acordo com a etiologia - em congênitas e adquiridas - e localização - em diafragmáticas, intercostais ou cervicais. As adquiridas correspondem a maior parte, estando frequentemente associadas à traumatismo torácico ou cirurgia torácica prévios. O diagnóstico baseia-se na história clínica e exame físico, sendo confirmado por radiografia ou tomografia computadorizada de tórax. A abordagem de tais pacientes pode ser cirúrgica ou conservadora, levando em consideração aspectos clínicos. Esse trabalho relata o caso de uma paciente de 44 anos, com história de traumatismo torácico e drenagem intercostal prévios, que evoluiu, após quatro anos, com hérnia pulmonar intercostal, além de uma revisão da literatura sobre o tema.

Descritores: Endometriose. Leiomioma. Biomarcadores Tumorais.

\section{ABSTRACT}

The occurrence of pulmonary hernia is uncommon. According to Morel-Lavalle, they can be classified, according to their etiology - congenital and acquired - and location - into diaphragmatic, intercostal or cervical. The acquired ones correspond to most, being frequently associated with previous thoracic trauma or thoracic surgery. The diagnosis is based on clinical history and physical examination, confirmed by chest radiography or computed tomography. The approach of such patients can be surgical or conservative, taking into account clinical aspects. This work reports the case of a 44-year-old patient, with a history of previous chest trauma and intercostal drainage, who evolved, after four years, with intercostal pulmonary hernia, in addition to a literature review on the subject.

Keywords: Endometriosis. Leiomyoma. Biomarkers, Tumor.

\section{INTRODUÇÃO}

A ocorrência de hérnia pulmonar, ou pneumocele, é incomum ${ }^{1,2}$. Por definição, caracteriza-se pela protrusão do parênquima pulmonar revestido pela pleura visceral e parietal além dos limites da caixa torácica, para a parede torácica, mediastino ou diafragma ${ }^{3,4}$. O primeiro caso foi descrito no ano de 1.499 e, desde então, são encontrados cerca de 300 relatos na literatura mundial5,6.

Para o correto diagnóstico da hérnia pulmonar, é essencial compreender sua fisiopatologia e associá-la à história clínica e fatores de risco, complementando o estudo com métodos de imagem. A apresentação clínica, em sua maioria, é assintomáti$\mathrm{ca}^{7}$. Quando presentes os sintomas mais prevalentes são dor torácica e dispneia, além de tumoração palpável na parede torácica ${ }^{8}$. As etiologias são variadas, podendo ser congênitas ou adquiridas (estas subdividem-se em espontâneas, traumáticas, patológicas e pós-cirúrgicas) ${ }^{8}$. Atualmente, as condutas em relação ao tratamento são baseadas apenas em relatos de caso, dada a raridade da doença em questão ${ }^{5}$. Em sua maioria, as abordagens cirúrgicas são preferidas ${ }^{5}$.

Apesar das hérnias intercostais serem raras e, em sua maioria, benignas, é importante seu conhecimento pelos médicos e profissionais da saúde para que, diante de um quadro característico, saibam como proceder e evitar as iatrogenias.

\footnotetext{
1Universidade Federal de Juiz de Fora- Faculdade de Medicina - Departamento de Cirurgia/Cirurgia Torácica - Juiz de Fora, MG, Brasil.

2 Universidade Federal de Juiz de Fora - Faculdade de Medicina - Juiz de Fora, MG, Brasil.
} 
O objetivo deste trabalho é contribuir para a divulgação de tal doença, por meio do relato de um caso clínico de uma paciente portadora de hérnia pulmonar intercostal, tratada no Serviço de Cirurgia Torácica do Hospital Universitário da Universidade Federal de Juiz de Fora (HU-UFJF), discutindo seu diagnóstico, tratamento e revisão literária acerca do tema, além de elucidar a importância do mesmo para a prática diária do médico.

\section{RELATO DO CASO}

Paciente sexo feminino, 44 anos, tabagista 35 anos-maço, asmática, obesidade grau I (IMC $32 \mathrm{~kg} / \mathrm{m} 2$ ). Relata trauma torácico contuso há quatro anos em acidente automobilístico, com necessidade de drenagem torácica em selo d'água em hemitórax direito para o tratamento de pneumotórax. Encaminhada ao serviço de cirurgia torácica do Hospital Universitário da Universidade Federal de Juiz de Fora (HU-UFJF) com queixa de dispneia, tosse produtiva e dor torácica ventilatório-dependente, com piora noturna. Relata agravamento do quadro há 5 meses, quando sofreu queda da própria altura sobre hemitórax direito. Ao exame físico, saturação periférica de oxigênio de 98\% em ar ambiente e ausculta pulmonar sem alterações. Foi observado abaulamento da parede torácica à direita ao realizar manobra de Valsalva. Realizada tomografia computadorizada de tórax (Figura 1), que evidenciou defeito da musculatura do serrátil anterior e intercostal, entre quinto e sexto arcos costais, com herniação de parte do lobo pulmonar médio no pertuito formado, com colo medindo $3,9 \mathrm{~cm}$ e saco herniário medindo $5,3 \times 4,3 \times 3,5 \mathrm{~cm}$.

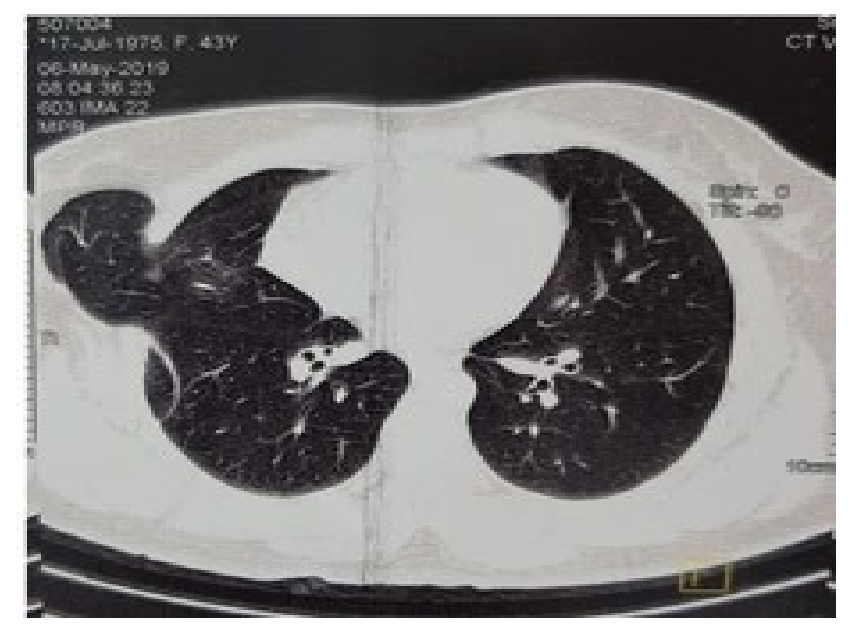

Figura 1. Tomografia Computadorizada de tórax evidenciando herniação do parênquima pulmonar.
Além disso, observou-se sequelas de fraturas consolidadas no quinto e sexto arcos costais direitos. Com os achados, foi estabelecido o diagnóstico de hérnia pulmonar intercostal à direita.

Em virtude da sintomatologia do quadro, foi indicado tratamento cirúrgico, com toracotomia na qual houve identificação da herniação (Figura 2), ressecção do saco herniário e intercostorrafia com pontos em "X" utilizando fio de PDS 1, com colocação de dreno torácico. Evoluiu sem complicações no pós-operatório, relatando apenas dor no local da incisão cirúrgica, com favorável controle após prescrição de analgésicos. A paciente encontra-se com 12 meses de evolução pós operatória, com ausência dos sintomas e de sinais sugestivos de recidiva do quadro.

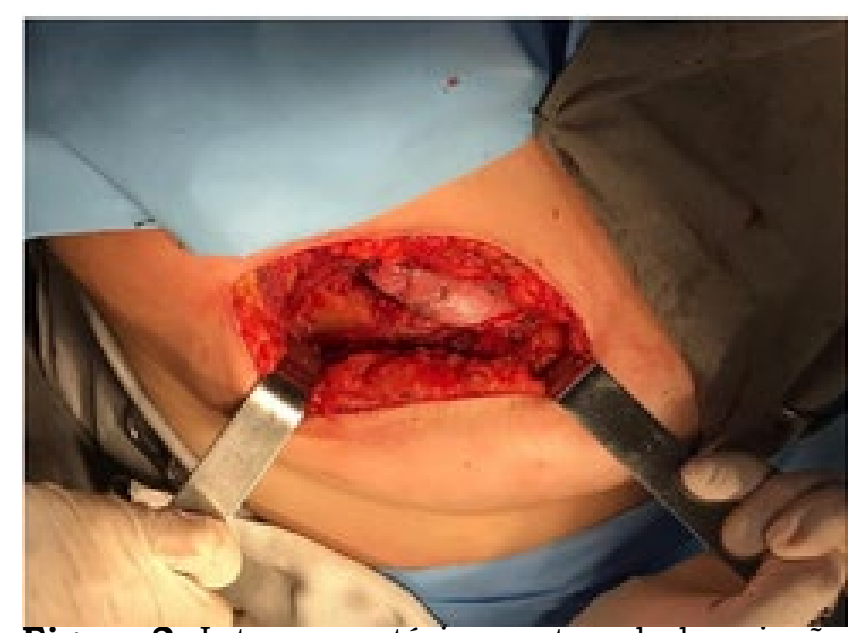

Figura 2. Intra-operatório mostrando herniação pulmonar através do quinto espaço intercostal direito.

\section{DISCUSSÃO}

As hérnias pulmonares são classificadas, segundo Morel-Lavalle (1845), de acordo com sua etiologia e localização anatômica 7 . De acordo com a localização, podem ser cervicais, torácicas ou diafragmáticas $^{7}$. Segundo a etiologia, dividem-se em congênitas ou adquiridas ${ }^{8}$. As hérnias congênitas são mais raras, correspondendo a $20 \%$ dos casos, enquanto as adquiridas representam $80 \%$ das hérnias pulmonares 9 . As hérnias adquiridas subdividem-se, ainda, em espontânea, patológica (secundária a neoplasias ou doenças inflamatórias), pós-cirúrgica ou pós-traumáticas. A hérnia do caso em discussão classifica-se, portanto, como hérnia pulmonar intercostal adquirida, podendo ser pós-cirúrgica (secundária à drenagem torácica) ou pós- 
traumática (em consequência de trauma torácico em acidente automobilístico ou queda).

As hérnias pulmonares adquiridas espontâneas estão associadas ao aumento da pressão intratorácica. Portanto, fatores que aumentem essa pressão estão diretamente relacionados ao maior risco de sua ocorrência, como obesidade, tosse crônica e estágio avançado de doença pulmonar obstrutiva crônica 1 . Ademais, há aumento do risco relacionado a causas de má cicatrização ou fraqueza tecidual, tais como diabetes mellitus, desnutrição, uso de corticoides ${ }^{10,11}$. Nos casos de hérnias pulmonares pós-traumáticas, costuma haver fratura de arcos costais associada, com a consequente lesão da pleura parietal subjacente ${ }^{12}$. As hérnias pós-traumáticas podem apresentar-se imediatamente após a lesão ou até anos depois ${ }^{3}$. As hérnias pós-cirúrgicas, por sua vez, estão mais associadas a procedimentos menos extensos, tais como minitoracotomias, em comparação com procedimentos torácicos maiores ${ }^{2,13}$. No caso relatado, a paciente possuía como fatores de risco obesidade, tabagismo de longa data, asma e tosse, além de histórico de trauma torácico, com fratura de arcos costais, e pequeno procedimento cirúrgico torácico (drenagem pleural fechada).

As hérnias congênitas frequentemente estão associadas a uma alteração subjacente na parede torácica e são resultantes de um defeito na fáscia de Sibson'9,12. Existem apenas dois relatos na literatura de hérnia pulmonar congênita isolada ${ }^{9}$.

A etiologia das hérnias intercostais pode ser explicada pela anatomia dos espaços intercostais. Existem três músculos que cobrem sua extensão: o músculo intercostal externo, interno e íntimo, sendo que suas distribuições incompletas dentro dos espaços leva a áreas de potencial fraqueza ${ }^{1}$. A borda anterior do espaço intercostal (adjacente ao osso esterno) e a borda posterior (adjacente à coluna vertebral) são as áreas de maior vulnerabilidade, pois são cobertas por apenas um desses três músculos ${ }^{14}$. Além disso, os espaços intercostais apresentam perfurações por onde passam os feixes vásculo-nervosos através da parede torácica ${ }^{2}$. Em consequência desses fatores, tais locais constituem os pontos mais suscetiveis às situações de aumento da pressão intratorácica e, consequentemente, herniação pulmonar. A localização do saco herniário geralmente é na parede torácica anterior, especialmente nos espaços intercostais inferiores (como no caso descrito, com herniação entre o quinto e o sexto arcos intercostais), seguido da posição lateral, sendo mais rara a ocorrência na região posterior. Isso também pode ser explicado pela anatomia, uma vez que a parte inferior anterior do tórax possui menos suporte muscular, e as cartilagens costais inferiores são mais amplamente separadas que as demais ${ }^{1}$.

O quadro clínico é variável. Uma parcela significativa dos pacientes encontra-se assintomático no momento do diagnóstico ${ }^{7}$. Quando existem sintomas, costuma-se notar dor torácica, dispneia e, em alguns casos, tosse ${ }^{13}$. Um estudo com revisão de 16 casos mostrou que a equimose foi observada em 44\% dos pacientes, sendo a terceira manifestação mais comum, após abaulamento $(100 \%)$ e dor no local $(87 \%)^{10}$. Ao exame físico, o achado de tumoração localizada, com tamanho que varia com a respiração e que piora à manobra de Valsalva é fortemente sugestivo do diagnóstico de hérnia pulmonar, além da dispneia, dor ventilatório-dependente e tosse ${ }^{6,15}$. As complicações mais frequentes identificadas na literatura consistem na dor crônica, na infecção pulmonar recorrente, no encarceramento ou estrangulamento pulmonar e na insuficiência respiratória, nenhuma delas presentes no caso estudado ${ }^{12}$.

A certeza diagnóstica depende de exames de imagem complementares. No caso da hérnia pulmonar, pode-se solicitar uma radiografia de tórax ou uma tomografia computadorizada de tórax (TC), método escolhido para avaliação do caso discutido ${ }^{2}$. A TC é um exame com alta sensibilidade para o diagnóstico e fornece dados importantes como localização fidedigna, informações sobre o orificio do saco herniário, características do parênquima herniado, tamanho da lesão e relação com parede torácica $^{2,11}$. Alguns autores sugerem a realização de TC concomitante à manobra de Valsalva, para aumentar a sensibilidade do exame $^{2}$.

O tratamento ainda é controverso, uma vez que a literatura carece de estudos com elevado nivel de evidência científica, em grande parte decorrente da raridade desta entidade clínica e motivo pelo qual é importante descrever relatos de manejo exitoso de hérnias intercostais ${ }^{9,12}$. 
A decisão terapêutica deve ser individualizada, levando em consideração o estado geral do paciente, a presença concomitante de doenças subjacentes, sintomatologia associada, as dimensões do defeito anatômico e o grau de viabilidade do tecido herniado 2,12 . Casos assintomáticos, de pequeno tamanho, não encarcerados, podem ser abordados de forma conservadora, com acompanhamento clínico 2,13 . Nos casos onde há sintomatologia, como o que se descreve acima, ou complicações (encarceramento, infecção, hemoptise, aumento de tamanho), o tratamento cirúrgico está indicado $^{9}$. O objetivo do tratamento cirúrgico é o fechamento do defeito intercostal, o que pode ser realizado a partir da rafia primária do defeito ou uso de material sintético ou autólogo - em geral nos casos em que há maior protrusão ${ }^{11}$. Quando realizado o tratamento cirúrgico, o procedimento aberto geralmente é executado. No entanto, atualmente a videotoracoscopia está ganhando mais espaço ${ }^{3}$. Alguns autores defendem o tratamento precoce, pois acreditam que as complicações potenciais de não reparar a hérnia sejam maiores do que aquelas associadas ao reparo ${ }^{15}$. Nossa escolha foi por toracotomia com rafia primária, levando-se em consideração o estudo pré-operatório realizado sobre o caso. O prognóstico é favorável, sendo, no geral, uma condição benigna. A evolução do caso em questão cursou sem complicações e recidivas, ilustrando a benignidade desta afecção quando há correta condução terapêutica da hérnia pulmonar.

O estudo possui a limitação por se tratar de um relato de caso. No entanto, dado a raridade do caso, consideramos fundamental contribuir com uma revisão que possa ajudar ao cirurgião na condução destes casos.

\section{CONCLUSÃo}

O correto manejo dos pacientes com hérnia pulmonar deve considerar fatores epidemiológicos, clínicos e radiológicos que, quando bem avaliados e associados à conduta terapêutica adequada, fornecem um prognóstico favorável ao paciente.

\section{REFERÊNCIAS}

1. Scelfo C, Longo C, Aiello M, Bertorelli G, Crisafulli E, Chetta A. Pulmonary hernia: Case report and review of the literature. Respirol Case Rep. 2018; 6(8):354.

2. Zapata R, Botero JE, Bolivar L, Saldarriaga C. Hernia pulmonar espontánea secundaria a acceso de tos; reparación por técnica asistida por vídeo. Rev Colomb Cir. 2015;30:2925.

3. Memon AS, Khan NA, Asghar FA. A case report of thoracic pulmonary hernia. Saudi J Health Sci. 2016; 15(2):97-9.

4. Karlen H, Spina JC. Hernia pulmonar post-traumática. Rev Am Med Respir. 2017;17(1):92-3.

5. Silva SRC, Carneiro F, Silva JB, Rocha M, Pinto-de-Sousa J. Hérnia pulmonar intercostal encarcerada em contexto de trauma toracoabdominal. Relato de caso e revisão da literatura. Rev Port Cir. 2017;41: 41-6.

6. Gómez PE, Ferreiro S, Esteban SM, Fodor R, Palavecino ME, Rios FG. Spontaneous Pulmonary Hernia: A Case Report. Respir Care. 2013, 58(10):119-22.

7. Marsico GA, Boasquevisque CHR, Loureiro GL, Marques RF; Clemente AM. Hérnia traumática do pulmão. Rev Col Bras Cir. 2011;38(1):77-8.

8. Serna M, Alba MAM, Alarza FH, Martínez RJA, Fernández MCM, Pardo JCM. Hernia pulmonar intercostal espontánea secundaria a cuadro de tos. Rev Patol Respir. 2012;15(3):8991.

9. Tapsoba TW, Grapin-Dagorno C, Bonnard A, El-Ghoneimi A. Isolated congenital inter-costal pulmonary hernia: a case report. J Med Case Rep. 2019;13(1):232.

10. Maeda T, Sato R, Luthe SK, Russell, MC. Spontaneous Intercostal Lung Hernia. Am J Med. 2017;130(9):399400.

11. Sanjuanelo SL, Sanz IL, Jiménez JZ, Valiente BA, Elena JMI. Hernia pulmonar espontánea: presentación de 4 casos y revision de la literatura. Cir Esp. 2017;95(4):237-9.

12. Bagenal JD, Capoun R, Casali G. Lung hernia following minithoracotomy for epicardial lead placement. BMJ Case Rep. 2013;2013:bcr201 3009317. 
13. Davakis S, Syllaios A, Mpaili E, Sdralis E, Charalabopoulos A. Minimally invasive oesophagectomy and emerging complications: intercostal lung hernia. Ann $\mathrm{R}$ Coll Surg Engl. 2020;102(3):73-4.

14. Weissberg D, Refaely Y. Hernia of the lung. Ann Thorac Surg. 2002; 74(6):1963-6.

15. Fine J,Walters BS, Agnoni AA, Coppola CP, Scorpio RJ, Kennedy AP. Pulmonary Hernia in a Two-Year-Old Child. Case Rep Surg. 2014; 2014:792376.
Recebido em: 27/05/2020

Aceito para publicação: 04/06/2020

Conflito de interesses: Não

\section{Endereço para correspondência:}

Marcus da Matta Abreu

E-mail: marcusdiretoria@hotmail.com gabriel3p@hotmail.com 